\title{
Unpacking Intuition: A Process and Outcome Framework
}

Julie Gore and Eugene Sadler-Smith*

School of Management, University of Surrey, UK

Accepted for publication Review of General Psychology 2011

*Address for correspondence: Professor E. Sadler-Smith, School of Management, University of Surrey, Guildford, UK. GU2 7XH.

$+44(1) 483683101$

e.sadler-smith@surrey.ac.uk 


\begin{abstract}
In recent years the topic of intuition has become an important focus of attention in psychology. It is often assumed to be a unitary construct however recent research suggests that intuition is multi-faceted. This paper disaggregates intuition by: discriminating between domain-general mechanisms and domain-specific processes of intuiting, and primary types of intuition and secondary types of intuition. The theoretical relationships between and within processes and types are examined and analyzed at behavioral and information processing levels, noting the importance in advances in social cognition research. As a result of this analysis we provide a conceptual framework which connects intuitive processes and outcomes. The paper concludes by outlining some of the implications of the framework and in particular highlights future methodological challenges faced by intuition researchers in laboratory and organizational field settings.
\end{abstract}

Key words: dual processing; intuition; information processing; social cognition 
Unpacking Intuition: A Process and Outcome Framework

Recent years have witnessed an upsurge in intuition research (e.g. Plessner, Betsch, \& Betsch, 2008; Glöckner \& Witteman, 2009). Despite many notable advances, researchers have tended to treat intuition as though it were a unitary construct (e.g. Ambady, 2010; Salas, Rosen, \& DiazGranados, 2010). This conceptual problem has not gone un-noticed. Hogarth (2010) stressed the need to identify types of intuition, classify the different mechanisms, specify their functions, and thereby "illuminate the conditions under which they are functional and dysfunctional for the organism" (p.343). Doing so has proved to be a theoretical and empirical challenge. Some writers on the subject have proceeded on an atheoretic basis offering descriptive and / or prescriptive accounts of intuition in different spheres of personal and professional life (e.g. Duggan, 2007) whilst others approached the subject from a non-psychological perspective (e.g. Crossan, Lane, \& White, 1999). In the organizational behavior field Allinson and Hayes (1996) chose not to 'unpack' intuition, instead they subsumed it under a general unitary construct of cognitive style. Within social psychology Pacini and Epstein (1999) attempted to disaggregate intuition by distinguishing between intuitive ability and intuitive engagement. Other researchers have sought to conceptualize different types of intuition in terms either of intuitive outcome or intuitive process (e.g. Dane \& Pratt, 2009; Glöckner \& Witteman, 2010; Sadler-Smith, 2008, 2010; Sinclair, 2010). Notwithstanding the various advancements that have been made in this regard a more integrative approach is now needed which seeks to encompass both outcomes and processes. In this paper we provide a new framework which aims to account for the multi-faceted nature of intuition and connects both intuitive processes and outcomes, see Figure 1. 
We begin the paper by providing a defense for pluralism in intuition by exploring why intuition is not a unitary concept. Next we examine distinctions between domain-general and domain-specific mechanisms of intuition. We continue by noting primary and secondary types of intuition and recent developments in social cognition. Thus, our new framework serves to more holistically understand the concept of intuition by bringing together disparate and seemingly contradictory areas of empirical work, highlighting previously unacknowledged connections.

[FIGURE 1 ABOUT HERE]

\section{Intuition is not unitary}

Many behavioral and brain scientists accept that two separate systems underlie human thinking and reasoning (see Stanovich \& West, 2000). It must be acknowledged there are however, notable exceptions to this interpretation; for example a number of behavioral and neuro-scientists are now somewhat skeptical of the dual-systems approach and alternatives have been advanced. For example, Kruglanski and Orehek (2007) proposed a 'quad' model, whilst others have suggested a multi-systems approach focused on the determination of value and subsequent choice (see Kable \& Glimcher, 2009 for a review). Nonetheless the dualistic partition between modes or systems represents "the dominant conceptual approach" (Kruglanski \& Orehek, 2007, p.307). Within many dual-processing frameworks System 1 thinking and reasoning is hypothesized as evolutionarily the more ancient of the two systems (Evans, 2003), its core processes are rapid, parallel, and automatic, permitting judgment in the absence of conscious reasoning (Epstein, 1994; Stanovich \& West, 2000); System 2 is more recent, its core processes are slower, serial, and effortful, permitting conscious abstract reasoning and hypothetical thinking (see Bargh \& Chartrand, 1999; Evans, 2008; Schneider \& Shiffrin, 1977; Wilson, Lindsay, \& Schooler, 2000). To date intuition has been identified 
as a System 1 process (Stanovich \& West, 2000). The aim of this paper is to elaborate on the claim that "further useful distinctions [are] to be made within the two processes of dual models" (Hogarth, 2010, p.342 italics added) on the basis that System 1 "contains many subsystems" (Hogarth, 2001, p.209) and that it is "almost certainly wrong to think of System 1 as one system" (Evans, 2008, p.17). Indeed Epstein, Pacini, Denes-Raj and Heier (1996, p.403) postulated that just as mathematical, verbal and abstract logic comprise rational processing (i.e. System 2), there may also be "several experiential [System 1] abilities, such as visualization, imagination, and aesthetic sensibility" (i.e. System 1). Thus, the unpacking of intuition to an extent echoes the multi-systems approach emerging within neuroscience.

The idea of intuition as non-unitary is not new, for example Wild (1938) distinguished between aesthetic, moral, and religious intuitions, but the usefulness of a number of the older classifications is now doubtful (e.g. Cappon, 1994; Vaughan, 1979). Also, Dane and Pratt (2007) noted that a confusing aspect of intuition research has been a tendency to call intuitive processes and associated outcomes 'intuition', they suggested that intuition is better conceptualized in terms of its process (the mechanisms and processes of intuiting) and its outcome (types of intuition). Table 1 outlines a further refinement of this distinction, i.e. within intuiting (domain-general mechanisms and domain-specific processes), and between intuitions (primary types and secondary types).

[TABLE 1 HERE]

\section{Domain-general mechanisms of intuiting}

Accepting that "intuitions are affectively charged judgments which arise rapidly through non-conscious, holistic associations" (Dane \& Pratt, 2007, p.40) three domaingeneral mechanisms of intuiting are posited: the application of heuristics under conditions of uncertainty; the acquisition and activation of complex domain-relevant schemas; the infusion 
of affect into decision making. The distinction between domain-generality and domainspecificity in the context of intuition draws on Chiappe and MacDonald's general set of assertions: (1) without domain-general mechanisms humans would be unable to solve recurrent problems in novel ways; (2) domain-specific mechanisms are insufficient of themselves; (3) domain-general mechanisms are central to human cognition, and necessary in order for human beings to be able to "cope with life in a constantly changing world" (2005, p.10).

This stance towards domain-generality assumes that there are certain preset goals that System 1 was designed, by evolution, to solve. Hence, humans possess evolved motivational systems that "provide positively or negatively valenced [affective] signals" that help to solve problems by narrowing down the array of possible solutions in situations where there may be a large number of behavioral choices (including solutions that worked well in the past) through automatic, unconscious processing (see Chiappe \& MacDonald, 2005, p.7).

First we examine heuristics as a source of errors and biases under conditions of uncertainty. We concur with the view that systematic biases accrue in human judgment as a result of a number of fallacies and intuitive errors in information processing (Kahneman, Slovic, \& Tversky, 1982). Intuitive judgments - at least in the heuristics and biases tradition are "thoughts and preferences that come to mind quickly and without much reflection" occupying a position between the automatic operations of perception and the deliberative operations of reasoning (Kahneman, 2002, p.449). Later developments in this program of research highlighted that a number of important biases and framing effects in judgment and decision making accrue from an affect heuristic (Slovic, Finucane, Peters, \& MacGregor, 2004). Finucane, Alhakami, Slovic, and Johnson (2000) offered evidence from experimental studies for an inverse relationship between perceived risk and perceived benefits based on the role of affect in judging the risks and benefits of specific hazards (e.g. nuclear power). Under 
time-pressured conditions reliance on affect was enhanced; for example, providing information designed to alter the favourability of participants' overall affective evaluation of nuclear power systematically changed risk/benefit judgments. Finucane et al. (2000) concluded that people draw intuitively on an affect heuristic which improves the efficiency of judgments by deriving risk/benefit evaluations from a common source, i.e. their 'gut feel' reactions. Slovic and colleagues asserted that in intuiting individuals draw on an affect pool containing "all the positive and negative [affective] tags associated with the representations consciously or unconsciously" (Finucane, et al., 2000, p.3). The affect heuristic confers irrationality on some aspects of judgment, but it can also offer strong advantages particularly in relation to interpreting subtle social cues communicating tacit knowing (De Martino, Kumaran, Seymour, \& Dolan, 2006).

Evolutionarily, heuristics are adaptive for survival in relevant ancestral environments (e.g. the concepts of Homo heuristicus and 'the adaptive toolbox', Gigerenzer \& Brighton, 2009) but are an ineffective means for dealing with statistical and probability-based problems (e.g. Wason selection task) in modern environments (Lakoff \& Johnson, 1999). Certain types of heuristics may be seen as a computational malfunction attributable to an inherent limitation of System 1 processing which operates maladaptively under particular conditions in non-ancestral (i.e. present day) environments. An alternative view is that heuristics are neither irrational nor a-rational, they are 'natural assessments' and "sensible estimation procedures" based on sophisticated underlying processes (e.g. retrieval and matching) in response to fairly simple questions posed under conditions of uncertainty, rather than assessments for complex judgmental problems (Gilovich \& Griffin, 2002, p.3). Indeed Gilovich and Griffin are keen to point out that in the heuristics and biases tradition decision makers are seen typically through a 'cognitive miser' lens (i.e. humans as conservators of 
mental effort). A natural assessments view ought to be consolidated as being "what the heuristics and biases program is really about" (2002, p.16).

Second, we explore complex domain-relevant schemas (CDRS) as bases for intuitive judgment under conditions of complexity and/or time pressure: System 1 processing is autonomous (its execution is rapid and mandatory when triggering stimuli are encountered), is not under conscious control or dependent on concurrent inputs from the analytic system (Toplak, Liu, Macpherson, Toneatto, \& Stanovich, 2007). System 1 processing is triggered by, and enables the solution of, problems that have recurrent features and involves deployment of complex domain-relevant schemas (CDRS) (Klein, 1993, Klein, et al., 1995). CDRSs are comprised of a web of declarative and procedural knowledge acquired through learning and supported by practice and feedback in environments conducive to the development of effective performance (Dane \& Pratt, 2007; Hogarth, 2010; Lipshitz, Klein, Orasanu, \& Salas, 2001). For individuals to develop the requisite CDRSs in occupational domains they need to engage in deliberate, motivated, and repetitive practice over the longterm (Ericsson \& Charness, 1994) in simulated and field settings with expert feedback that allows them to "learn the appropriate lessons from experience" in 'kind' learning environments (Hogarth, 2001, p.90). Individuals may acquire such knowledge through exposure to prototypes, observational learning (Epstein, 2008) and social interaction, even though they may not be able to articulate the tacit knowledge and implicit understanding acquired (Nadler, Thompson, \& van Boven, 2003). Based on a summary of current models of intuition Glöckner and Witteman (2010, p.1) proposed a "categorization according to the underlying cognitive processes" of four mechanisms of learning, retrieval, and integration which support the role of CDRSs in intuiting, namely: associative (including classical and operant conditioning); matching (including the acquisition of prototypes); accumulative (including accumulation of evidence based on quick automatic processes such as matching); 
constructive (including automatic construction of mental representations and coherence shifts).

The acquisition of CDRSs is pertinent not only to the problem-solving intuitions typical of expert performance, also prolonged exposure and learning in a specific sociocultural setting may produce an intuitive facility for moral or social judgment as product of domain-general learning mechanisms (Almor, 2003). For example, Sonenschein (2007) argued that managers with little experience in arriving at moral judgments may have few intuitions about ethical issues, but as a result of exposure and experience (and the acquisition of moral and ethical prototypes) over the longer term they become more able to rely on intuitions which have captured past experiences. An implication of this is that unethical behaviors in organizations may be more typical of novices rather than experts, the latter are likely to have acquired well-honed virtues whereas the former "struggle with applying the right rule in [complex and ambiguous] situations requiring many rules" (Sonenschein, 2007, p.1033).

Third, we note somatic markers as sources for the infusion of affect under conditions of risk as a third possible domain-general mechanism of intuiting. Affect infusion is a process whereby "affectively loaded information exerts an influence on, and becomes incorporated into, cognitive and judgmental processes" (Forgas, 2001, p.101). The somatic marker hypothesis (SMH) (Damasio, 1999) provides a systems-level neuro-anatomical account for the infusion of affect into risk-based decision-making the mechanisms of which occur at a non-conscious level. The SMH is posited on the principle that "decision-making is a process that is influenced by [somatic] marker signals that arise in bio-regulatory processes, including those that express themselves in emotions and feelings" (Bechara \& Damasio, 2005, p.336). 
The origins of the SMH may be traced to experiments that compared the performance on a simulated high-risk gambling task of normal participants with that of patients with damage to the ventro-medial prefrontal cortex (VMPC, implicated in the induction of emotions). The essence of the SMH is that a pattern of somatic and visceral signals from the body act as marker signals (a warning signal when negatively valenced) and guide (e.g. by narrowing) decision-making in advance of conscious awareness based upon previous experiences (Bechara, 2004). On the basis of Dane and Pratt's (2007) definition of intuition (i.e. it is 'affectively charged') such autonomous 'narrowing signals' may themselves be classified in terms of the valence of the associated affect (positive or negative, signaling 'approach' or 'avoid' behaviors respectively) and intensity, (high ['hot'] or low ['cold']), i.e. 'hot approach', 'hot avoid', 'cold approach', and 'cold avoid' gut feelings (see Metcalfe \& Mischel, 1999). Indeed Haidt (2004) has argued that it is incorrect to contrast affect and cognition, preferring instead two kinds of cognition "hot and cold" (p.286). Somatic markers can serve as a neurobiological 'alarm' mechanism or a 'beacon' of incentive (which may or may not be consciously apprehended) in the selection of 'good' responses over 'bad' ones in terms of "survival versus [exposure to] danger" (Damasio, 1996, p.1417) thereby sustaining adaptive avoid/approach behaviors. For a critical review of the SMH see Dunn, Dalgleish, and Lawrence (2006).

We now proceed with a discussion of how our framework encapsulates primary types of intuition (i.e. the outcomes of the process of intuiting).

\section{Primary types of intuition}

The mechanisms of intuiting described above are domain-general - they operate automatically across domains on the basis of the complexity, uncertainty, and level of risk associated with a triggering event. There are also a number of specific processes which 
pertain to particular domains ('domain-specific processes') see Table 2. We discuss four intuition-relevant domains: problem-solving, creativity, moral judgment (Dane \& Pratt, 2009; Haidt, 2001; Klein, 2003; Reynolds, 2006; Sinclair, 2010; Sonenschein, 2007), and social judgment (Almor, 2003; Ambady, 2010; Myers, 2001). These recur across a wide variety of occupational and non-occupational settings. Each primary type (as distinct from secondary types, see 'Concluding Remarks' below) will be described and analyzed in terms of: definition; behavioral description; domain-specific information processing mechanisms; neural correlates - see Table 2.

[TABLE 2 ABOUT HERE]

\section{Problem-solving intuition}

Definition: Domain-specific, expertise-based response to a tightly-structured problem based on non-conscious processing of information, activated automatically, eliciting matching of complex patterns of multiple cues against previously acquired prototypes and scripts held in long-term memory.

Behavioral description: Dane and Pratt (2009) described this type of intuition as pattern matching "honed through repeated training and practice" $(2009$, p.5). Such intuition is deployed typically by experts in order to deal with tightly-specified problems in particular domains. Dane and Pratt (2009) refer to it as 'problem-solving intuition' rather than 'intuition-as-expertise' (Hogarth, 2001) or 'intuitive expertise' (Kahneman \& Klein, 2009). Problem-solving intuitions are triggered by tightly-structured decisions and problems requiring convergent solutions, for example, Naturalistic Decision Making (NDM) researchers such as Klein and his colleagues have studied complex, time pressured, highstakes decisions such as whether or not and how to evacuate a burning building, or if and how intervene in a medical emergency. 
In the 1980s Klein and his co-researchers developed a recognition-primed decision (RPD) model based on: (1) narrative accounts of complex, time-pressured decisions taken by experienced fireground commanders' which did not fit into explicit decision-tree, multiattribute, or optimal choice models; (2) the possibility that "fireground commanders were contrasting alternatives, but at an unconscious level" (Klein, 1993, p.139); (3) the deployment of intuition in environments in which there is inadequate information, where goals are unclear and procedures are poorly defined, where cue learning enables patterns to be perceived and distinctions to be made, and where conditions are dynamic (Klein, 2003).

The decision makers studied by RPD researchers, (for example, firefighters, neo-natal nurses, and missile battery commanders) operated in time-pressured, complex, life-or-death situations where there was little or no time to compute multiple options, therefore they used their experience to 'size-up' situations, get a sense of typicality, recognize a course of action that matched a prototype, and implement it. Effective intuitive problem-solving that is capable of yielding judgments that are consistent, valid and reliable over the longer term depend upon prior experience, learning, and feedback upon which the capacity for rapid response through recognition is built (Simon, 1987). However, RPD strategies are unlikely to be productive or necessary in computational-type tasks, where justifications are required and where the views of different stakeholders have to be taken into account (Lipshitz, Klein, Orasanu, \& Salas, 2001).

Information processing mechanisms. Based on research that had its inception in a series of small-scale experiments on the role of 'chunking' in chess and on a body of empirical evidence indicative that expert decision makers rely on a process of pattern recognition which is unavailable to novices (Simon \& Chase, 1973), Simon summarily described "intuition and judgment - at least good judgment..." as “...simply analyses frozen into habit and into the capacity for rapid response through recognition" (1987, p.63). 
Following in the tradition of Simon's seminal contribution NDM researchers are concerned with understanding and improving decision making as it pertains to complex judgmental tasks undertaken by experienced participants in field settings (as opposed to naïve participants in laboratory settings, Salas \& Klein, 2001; see also Gore et al, 2006), i.e. 'intuition-as-expertise' (Hogarth, 2001) or more latterly 'intuitive expertise' (Kahneman \& Klein, 2009).

Central to the RPD model are learned holistic associations based on prototypes that enable an experienced decision maker to gain an acute awareness of a situation based on relevant cues (compared to a novice who might devote scarce mental resources to irrelevancies), deploy action scripts (activated by meaningful patterns of cues based on similarity to previously-encountered situations), mentally simulate the deployment of a given script (based on mental models), take the necessary actions (based on requisite levels of skill), and accomplish the whole process "in an instant and without conscious thought" (Klein 2003, p.14). However, expertise and intuitions are not synonymous: "there are mechanisms of expert decision-making performance that involve intuitive processing and those that involve deliberative processing" (Salas, et al., 2010, p.10). Kahneman and Klein (2009) argued that the determination of whether or not an intuitive judgment can be trusted depends on the validities of task environments and participants' learning. High-validity environments are characterized by stable relationships between objectively-identifiable cues and subsequent events, or between cues and the outcomes of possible actions. The latter may often be the case in medicine and fire-fighting in situations involving expert participants, but not in predicting the future value of individual stocks, or long-term political forecasting (Kahneman \& Klein, 2009). High-validity environments allied to prolonged practice and timely and unequivocal feedback (Hogarth, 2001) are necessary for the development of expert (i.e. (well-informed') intuitions. 
Neural correlates: As far as the neural bases of pattern recognition are concerned, evidence is emerging from laboratory studies which may form the basis of an improved understanding of expertise-based intuition as it occurs in field settings. Inferences are tentative at this stage but provide foundations for further work. For example, the complex spatial representations deployed in expert navigation by London cab drivers was found to be associated with greater posterior hippocampal gray matter volume (Maguire, Woollett, \& Spiers, 2006). Campitelli, Gobet and Parker's (2005) study of expertise in chess "strengthen[ed] the hypothesis that when performing a domain-specific task experts activate different brain systems from that of novices" (p.238). Domain-specific information stored in the temporal lobes supports quick recognition (Campitelli, Gobet, Head, Buckley \& Parker, 2007). Bar, et al. (2006) suggested that the role of the brain's frontal cortex is to provide an initial 'guess' at recognition which serves to limit the number of options to be considered. This activation occurs prior to activation in the brain regions involved in object recognition. Such processes are advantageous to the extent that they enable a quick response to be generated based on an initial extraction of the 'gist' of a stimulus in situations characterized by time pressure and/or insufficient information. The costs of acting erroneously on the basis of such an initial judgment based on limited information are likely to be outweighed by the costs of not acting on such information at all, hence fast responses based on a crude 'gist extraction' are likely to have been adaptive in ancestral environments (see Le Doux, 1996). Volz and von Cramon (2006) used fMRI to study brain regions involved in intuitive judgments of patterns of visual coherence, and found that the orbito-frontal cortex (OFC), amygdala, and other brain regions, including the ventral occipito-temporal (VOT) regions, were activated autonomously in coherence judgments, even when participants were unable to explicitly name the stimulus. In summary: ‘upstream' processes may provide initial 
interpretations for use by 'downstream' brain regions (Volz \& von Cramon, 2006) in arriving at quick assessment via the extraction of the gist of a situation (see Brainerd \& Reyna, 1990).

\section{Creative intuition}

Definition. Slow-to-form affectively-charged judgment occurring in advance of an insight that combines knowledge in novel ways based on divergent associations, and which orients behavior in a direction that may lead to a creative outcome.

Behavioral description. Creativity involves the generation of new and valued ideas, and psychologists have been concerned traditionally with the cognitive processes leading up to idea generation. There is a body of narrative and experimental evidence which supports the view that creative breakthroughs are often presaged by an incubation phase in which intimations, and 'feelings of knowing' (Heli \& Sun, 2010; Koriat, 1993) are experienced in the lead-up to the moment of insight (Davidson, 1995). Hence, creative intuition is the subjective experience of phenomena often referred to as 'vibes', 'hunches' or 'gut feelings' which may intimate an impending insight. The role of such intimatory phenomena, which typically are positively valenced, is attested to by many well-documented examples in the history of scientific discoveries and technical advancements (Gruber, 1995; Sundgren \& Styhre, 2004) and artistic achievements (Claxton, 2001; Harvey, 1999).

One potential source of confusion relates to the fact that insight and intuition are not the same (Hogarth, 2010), however they are closely related most notably in the domain of creativity to the extent that a creative intuition segues to an explicit insight at the so-called 'eureka moment'. Creative intuitions provide a visceral sense interpreted as an intimation (literally 'an announcement') that a conjecture (such as a hypothesis, sketch, or plan) may work even though formal evaluation of its viability may be some way off (Gick \& Lockhart, 1995). Creative intuitions differ from problem-solving intuitions principally in that they are 
slower and the latter do not necessarily combine knowledge in novel ways, but instead rely upon convergence between an observed pattern and a prototype held in long-term memory (Salas et al., 2010). Hence, unlike problem-solving intuitions, the nature of associations in creative intuitions is divergent rather than convergent (Dane \& Pratt, 2009).

Creative intuitions, in which knowledge is combined in divergent, holistic, and novel ways support scientific discovery, technical invention, business venturing, and artistic endeavor (Claxton, 2001; Dorfman, Shames, \& Kihlstrom, 1996; Miller \& Ireland, 2005; Policastro, 1995). Creative intuitions are conjectures, they signal directions of divergent thinking that might turn out to be fruitful, but also which might be 'blind alleys' and in common with intuitive judgments more generally have their attendant uncertainties.

A number of theories of creativity have identified cognitive styles as an important variable "essential to creativity" (Kaufman \& Baer, 2009, p.87) and which influence different phases of decision processes (Hunt, Krzystofiak, Meindl, \& Yousry, 1989). For example, individual differences in Kirton's adaption-innovation (A-I) cognitive style are associated with differing levels of creativity (Goldsmith, 1994), while the Type I intellectual style identified by Zhang and Sternberg (2009) is defined as being holistic, intuitive, divergent, and creativity-generating. Individual differences in cognitive style moderate the relationship between intuitive processes and intuitive outcomes (Simonton, 1980) to the extent that CDRSs are a necessary but insufficient condition for creativity (for example, in business venturing creative entrepreneurs also need be in possession of relevant expertise - see Crossan, Lane, \& White, 1999), it is also necessary to be able to make divergent associations (Policastro \& Gardner, 1999) through styles of processing variously labeled as innovative (Kirton, 1976), intuitive (Allinson \& Hayes, 1996) or Type 1 (Zhang \& Sternberg, 2009). Recent empirical evidence shows that an intuitive cognitive style is positively associated with the creative aspects of entrepreneurship (Kickul, Gundry, Barbosa \& Whitcanack, 2009). 
Information processing mechanisms. Autonomous neural activations are implicated in the inter-related, but sometimes conflated, processes of incubation, insight and intuiting (Dorfman, et al., 1996). The origins of the notion of incubation are traceable at least as far back as Wallas' (1926) model of creativity and problem-solving which he based on studies of the narrative accounts of a number of scientific discoveries. The model comprises four sequential stages: preparation, incubation, intimation, and illumination, and is welldocumented elsewhere (Sternberg \& Davidson, 1995). From the perspective of analyzing creative intuition, the intimation stage of the model is most relevant: Wallas described it as transitory sensations presaging an impending insight, a "fringe consciousness of an association-train is in the state of rising consciousness which indicates that the fully conscious flash of success is coming" (1926, p.97).

Wallas' model is a classic view of unconscious incubation involving activations beneath the threshold of awareness which are slow and spreading (Dorfman, et al., 1996; Yaniv \& Meyer, 1987). Moreover, creative intuition is unique amongst types of intuition in being the result of relatively slow or suspended non-conscious processing of information (Claxton, 2006).

Neural correlates: Spreading activation across neural networks (Yaniv \& Meyer, 1987) and levels of activation achieving threshold levels such that they enter into conscious awareness (Bowers, Regehr, Balthazard, \& Parker, 1990) provide plausible neural level explanations for the phenomenon of creative intuition. Jung-Beeman, et al. (2004) mapped differences in neural activity between insight and non-insight problems, and observed that increased activations in the anterior superior temporal gyrus (aSTG) of the right hemisphere (RH) occurred at the moment of insight. The aSTG region facilitated the integration of information across wide semantic networks (enabling problem solvers to make remote connections through divergent, diffuse, and overlapping activations), whereas the neural 
circuitry in the same region of the left hemisphere produced narrower, more discrete fields of activation. Positive mood supported the spread of neural activation to remote associates in memory (promoting a holistic processing mode), whilst negative mood restricted the spread of activation (promoting a serial processing mode) (Bolte, Goschke, \& Kuhl, 2003). This is consistent with the finding that participants in positive moods demonstrated divergent thinking, made unusual associations and performed well on insight problems (George \& Zhou, 2007).

\section{Social intuition}

Definition. Rapid and automatic evaluation of another person's cognitive and/or affective state through the perception and non-conscious processing of verbal and/or nonverbal indicators.

Behavioral description. The ability to detect important social attributes of conspecies, for example their willingness as potential partners in valuable behaviors such as cooperation or mating, could confer reproductive advantage and therefore be more likely to spread through a population (Almor, 2003, p.105). This capacity to identify rapidly and automatically with the mental states, motives, feeling states, and intentions of others is described by Myers (2002, p.33) thus: "When meeting a stranger in the forest, one had to instantly assess whether that person was friend or foe", and individuals who were able to do so and act accordingly "were more likely to survive and leave descendents". Whether or not another individual is 'friend' (evoking a positively-valenced intuition signaling attraction) or 'foe' (evoking a negatively-valenced intuition signaling avoidance) presents a tightlystructured problem, and is an analogue for many social interactions and judgments that occur in occupational settings (such as selection interviews, negotiations, co-worker preferences, and group dynamics). 
Within the area of social psychology dual process models continue to be developed. Social psychologists such as Smith and DeCoster (2000) suggest that generally three components are evident: reports of how people process in a 'quick and dirty' fashion; how they process when they have completed extensive thought; and an exploration of what conditions facilitate effortless processing. Smith and DeCoster (2000) note that dual-process models in social and cognitive psychology overlap and they propose a new model which links these areas by examining underlying memory systems. They argue that the underlying dual processing modes are qualitatively different types of processing. For example, they note that stereotypes may be held in a slow-learning memory system in spite of people who may deny those beliefs. They suggest two processing modes which are 'associative' and 'rule-based' (Sloman, 1996; Smolensky, 1988) and draw on two different memory systems, something which further research on cognition may do well to consider (e.g. the relative merits of unconscious thought, Dijksterhuis, 2004).

The evolutionary and neural bases, and ontogenetic trajectories of 'hard-wired' social intuition are uncertain at present (Singer, 2006), but it is argued that a system of reasoning goals, perceptions, and emotions develops in the first two years of life (Saxe, Carey, \& Kanwisher, 2004), and that people can and do express implicit attitudes that are activated automatically the basis for which is unknown to them but which influence implicit responses as part of a dual-attitude system (Wilson, Lindsay, \& Schooler, 2000). As studies of implicit prejudice and intuitive fears illustrate (Myers, 2002), intuitive social judgments can be accurate but also are prone to errors and biases and may lead us astray (Kahneman \& Frederick, 2002; Nisbett \& Ross, 1980). Intuitive social judgments are accompanied by a relatively high level of affective charge (Adolphs \& Damasio, 2001), and the rules of inference are relatively closed to introspective access (Nisbett \& Wilson, 1977). In common with moral intuitions (see below), social intuitions are shaped by the socio-cultural context in 
which they develop. For example, Myers (2002, p.47) speculated that women are seemingly "more empathic, more sensitive to nonverbal cues, and more relational" than men, and that their higher levels of social intuition may be as a result of an interaction between genetic (as a result of evolutionary pressures) and socio-cultural factors (females are expected by certain cultures or societies to be more empathic and relational) (see Allinson \& Hayes, 2000). There have been mixed findings in relation to, for example in relation to the stereotype of ‘female intuition' (Allinson \& Hayes, 1996), but the extent to which this is a result of researchers failing to disaggregate intuition (i.e. treating as a unitary construct) warrants further investigation.

Information processing mechanisms. Empirical evidence for the processes underlying 'impressionistic' intuitive social judgments may be found in a number of related studies initiated by Ambady and her colleagues in the early 1990s (for a summary see Ambady, 2010, p.271). These researchers observed that ratings by complete strangers based on evaluative 'thin slices' (video clips between two- and ten-seconds in length) of teachers' non-verbal behaviors predicted with high levels of accuracy the ratings of the same teachers by students who had interacted with them substantially, these researchers also reported correlations between thin slice judgments and real-life criterion variables (Ambady \& Rosenthal, 1993). Other thin slice research has found that the effectiveness of sales managers (as measured by supervisors' evaluations and actual sales) can be assessed accurately using thin slices of the vocal channel of communication, and that thin slices generally are more valuable for assessing inter-personal rather than non-interpersonal task-related skills (Ambady \& Krabbenhoft, 2006). Other examples of correlations between intuitive thin slice judgments and criterion measures include: surgeons' rated as domineering on audio thin slices were more likely to have been sued for malpractice in the past (Ambady, La Plante, Nguen, Rosenthal, \& Levinson, 2002); physical therapists' who were rated on video thin 
slices as distancing themselves from patients (for example by not smiling or by looking away) were more likely to have clients whose physical and mental functioning showed longterm decline (Ambady, Koo, Rosenthal \& Winograd, 2002).

Social intuitions are difficult to consciously control, for example while it may be possible to consciously and effortfully manipulate the content of the verbal channel, states such as anxiety are communicated implicitly and effortlessly through tone, pitch, and gesture and are evaluated intuitively (DePaulo, 1992) (by contrast, skilled liars are able to manipulate the verbal and non-verbal channels of communication, Porter, et al., 2008). Lieberman (2000, p.111) described the intuitive encoding and decoding of mental states as a "compelling example of intuitive social action" with implications for the understanding of script-based inferences (based on culturally-agreed orders of events in social interactions), distributed decision making, automatic evaluations, and other social inference processes which are relatively closed to introspection. The nature of associations involved in intuitive social judgments is divergent and broad, based on the integration of multiple sources of information from multiple channels (Ambady \& Rosenthal, 1993; DePaulo, 1992).

Neural correlates: According to Adolphs and Damasio there is "something of a consensus that the amygdala is critical to the recognition of emotions from facial expressions" (2001, p.40). Lieberman (2005) has observed that current theories and methods in behavioral science cannot give a satisfactory account of why some automatic social judgments, such as thin slices, can be highly accurate while others are systematically inaccurate. In an attempt to understand the nature of intuition-based self knowledge Lieberman, Jarcho, and Satpute (2004) used functional magnetic resonance imaging (fMRI) to examine the neural correlates of intuition-based and evidence-based self-knowledge. Intuition-based judgements produced activations in "a network of neural structures called the $X$-system, involved in automatic social cognition" whereas evidence-based judgments 
"produced activations in a network called the $C$-system, involved in effortful social cognition and propositional thought" (p.421).

The information processing and neural mechanisms of the C-system (lateral prefrontal cortex, medial temporal lobe, and medial parietal cortex, see Lieberman, 2007) are considered to yield analytically reasoned judgments; whereas those of the X-system (amygdala, ventro-medial pre-frontal cortex (VMPC [cf. somatic marker hypothesis above]), and basal ganglia, see Lieberman, 2007) are considered to yield intuitive judgments. According to Reynolds (2006, p.740) the C-system's rule-based analysis and active judgment has the potential to intervene, exercise executive control over, and "micromanage" the Xsystem. Lieberman (2005) suggested that social cognitive neuroscience (SCNS) may be in a good position to "tease apart the different forms of automatic social cognition" (p.752) in terms of the workings of the X-system.

Other cognitive neuroscientific explanations for intuitive empathic perception and judgment include the neural mechanisms associated with Von Economo and mirror neurons. On the basis that visceral intuitive responses enable quick reactions in social situations characterized by high uncertainty, Allman, Watson, Tetreault and Hakeem (2005) theorized that Von Economo neurons (VENs, also referred to as 'spindle neurons'), which are found only in humans and great apes, emerge mainly after birth, and reach their adult number by age four years, play an important role in intuitive social judgment. VENs may relay fast assessments of complex social situations thus allowing quick evaluations and rapid adjustments of behavior. They may have been selected for as an adaptation to the increased gregariousness and intensity of social interactions that have characterized latter stages of human phylogeny, specifically a need to quickly and effortlessly judge others' levels of altruism, empathy, and trustworthiness (Allman, et al., 2005). A 'theory of mind' (ToM) enables an individual to "attribute independent metal states to self and others in order to 
explain and predict behavior" (Frith \& Happé, 1994, p.116). Lieberman argued that the "sense of experiencing other minds appears to recruit brain regions more closely tied with automatic and affective processes" (2007, p.265) and that the lateral temporal cortex in particular supports automatic and non-reflective aspects of ToM (2009, p.21). Other researchers have suggested that disorders such as autism ('mind blindness') may be related to failure of VENs to develop normally (Allman, et al., 2005).

Mirror neurons are found in the frontal cortices of humans and apes, and are involved in motor cognition - they fire in response both to performing an action (such as grasping) and to the action being observed, i.e. they show congruence between the visual actions they respond to and the motor responses they code (Rizzolatti \& Craighero, 2004). Evidence for the involvement of mirror neurons in emotional interpersonal cognition can be found in studies of mirror neuron activation in tasks relying on empathic abilities without task-related motor components (Schulte-Rüther, Markowitsch, Fink, \& Piefke, 2007). Mirror neurons have been posited as playing an important role in non-verbal communication between interaction partners and the intuitive judgment of the behavior, intentions, and experiences of others (Lieberman, 2007), but this has yet to be demonstrated empirically. The mirror neuron system (MNS) offers a promising account for the processes whereby humans perceive others empathically (being 'like me' in the eyes of the observer) and allow us to "use the same systems that process knowledge about self-performed actions, self-conceived thoughts, and self-experienced emotions to understand actions, thoughts, and emotions in others" (Oberman \& Ramachandran, 2007, p.310).

\section{Moral intuition}

Definition. Automatic, rapid, affect-based judgment made in response to an ethical dilemma, arrived at non-consciously, rationalized post-hoc, and relatively impervious to disconfirmation (Greene \& Haidt, 2002; Haidt, 2001; Sonenschein, 2007). 
Behavioral description. Contrary to traditional rationalist theories of moral judgment, the concept of moral intuition draws on Haidt's (2001) social intuitionist model (SIM) of moral judgment based on the precept that individuals act like 'intuitive moral attorneys' (Sonenschein, 2007) who search for confirmatory evidence for their initial intuitions (i.e. 'gut feel' reactions to a moral dilemma). The SIM is 'intuitionist' in that it states that "moral judgment is generally the result of quick, automatic evaluations (intuitions)" (Haidt, 2001, p.814). It is consistent with a view of moral judgment as involving non-conscious pattern matching accompanied by a relatively high level of affective charge, i.e. reasoning is a post-hoc attribution (Monin, Pizzaro, \& Beer, 2007) thereby creating an illusion of control (Dane \& Pratt, 2009; Sonenschein, 2007). In an experimental study using a deliberative ethical decision-making task Marquardt and Hoeger (2009) found that managers' implicit (intuitive) moral attitudes exercised an important influence on their decision-making; they concluded that complex and deliberative decision-making processes in the context of business ethics are significantly affected by implicit moral attitudes. Even though individuals may ascribe their moral judgments and behaviors to rationality, intuitionist theories suggest that "individuals first use intuitions and then use post-hoc (moral) reasoning” (Sonenschein, 2007, p.1027). These 'emotion-backed' concerns about social interactions (i.e. moral intuitions) are a result of evolution "equipping [Homo sapiens], and other primates, for an intensely social life" (Haidt, 2004, p.286). It should be noted however that the SIM has not been without its critics, for example Saltzstein and Kasachkoff (2004).

Haidt's SIM is social in that emphasizes the significance of social and cultural influences on moral judgment. Social intuitions are learned gradually and implicitly by observation and imitation within the custom complexes of their socio-cultural setting, the latter providing a cultural "front end" for the somatic marker hypothesis (Haidt, 2001, p.828). Hence, moral intuitions are expressed differentially according to the culture in which they 
occur, even though the underlying processes may be universal (Hauser, 2006; Mikhail, 2007). Dane and Pratt (2009), commenting in relation to the cultural interactionist aspects of intuitionist moral judgment, noted that 'smaller cultures' such as those of organizations can also shape the moral and ethical codes of their members. This can lead to individuals internalizing not only the moral values of a culture, but also the lack thereof, moreover members of organizations may also displace personal responsibility for their actions with behaviors commensurate with organizational or institutional norms and values (Sonenschein, 2007) despite a moral instinct to the contrary.

Information processing mechanisms. Hauser (2006) attributed the roots of human morality to a number of innate, instinctive, and intuitive moral principles and processes which evolved as result of increased gregariousness in the shift towards living in relatively large social groups due to environmental changes (see Mithen, 1996). The outcomes of moral intuiting express themselves differentially across cultures, and are linked inextricably to affect ('gut feel'), often manifest in an inability to explicate one's moral reasoning (socalled 'moral dumb-founding', Haidt, 2001). Thus moral judgment is more a matter of “emotion and affective intuition than deliberate reasoning" (Greene \& Haidt, 2002, p.517) governed by a universally-held sense of right and wrong (for example, Rawlsian principles of justice, or of fairness) the processes underlying which: (1) guide moral judgment automatically and rapidly but do not determine action; (2) are inaccessible to conscious awareness; (3) operate on real and imagined experiences; (4) require little or no instruction; (5) can be impaired as a result of damage to the neural circuitry which 'hard-wires' the moral instinct (Cushman, Young, \& Hauser, 2006). In summary, moral intuitions draw on culturally variable moral systems which operate on a universal set of principles but which are honed to a set of moral norms based on prototypes, largely inaccessible and unconsciously operative, and which vary between socio-cultural complexes (Hauser, 2006). 
Neural correlates: Emerging themes from the nascent field of 'neuro-ethics' suggest that preferences for certain normative approaches to ethical decisions are associated with particular structures and mechanisms in the human brain (including the medial orbito-frontal cortex, the amygdala, and the anterior cingulate) implicated in emotional processing (Salvador \& Folger, 2009). A preliminary functional neuro-anatomy of moral judgment suggests that no single brain region is devoted exclusively to it (Greene \& Haidt, 2002), nonetheless impaired moral judgment has been observed in patients who have suffered damage to the ventro-medial region of the prefrontal cortex (VMPC) (Young \& Koenigs, 2007). Normative moral judgment is also thought to involve interplay between the reflexive pattern-matching X-system (amygdala, VMPC, and basal ganglia), and the reflective, conscious reasoning C-system (Salvador \& Folger, 2007) identified by Lieberman, Jarcho, and Satpute (1994). In the case of moral intuition, the X-system functions on the basis of ethical or moral prototypes, described as "dynamic constructs holding descriptive, evaluative and prescriptive information in one configuration of neural network signals", and results in a match or mismatch which may be further processed by the C-system (Reynolds, 2006, p.793).

\section{Concluding remarks}

In this paper we have argued that intuition should be conceptualized as a multidimensional rather than a unitary construct comprised of a variety of general and specific mechanisms and processes, and primary types. The term 'primary type' is adopted here in order to distinguish problem-solving, creative, social, and moral intuitions from composite forms of intuition. The latter, which may be referred to as 'secondary' types, are analyzable in terms of primary types and are deployed in specific and non-recurrent (i.e. less frequently encountered) domains in occupational settings (e.g. business management, education, medicine, law, etc.). For example, in the field of business and management researchers have 
proposed 'entrepreneurial intuition' (Sadler-Smith, Hodgkinson, \& Sinclair, 2008) which is composite to the extent that it draws on: (1) creative intuitions for the generation of new ideas (Kickul, et al., 2009); (2) problem-solving intuitions for the judgment of the viability of a business venturing proposition (Blume \& Covin, 2010; Crossan, et al., 1999); (3) moral intuition in deciding whether or not to invest in a deal which may have an ethical component (Buchholz \& Rosenthal, 2005); (4) social intuition in deciding who and who not to transact with (Shane \& Venkataraman, 2000). A corollary of this argument is that further secondary types may manifest in other applied areas such as healthcare, medicine, education, and law.

The nature of intuition presents a particular set of methodological challenges for the documenting intuitive decision processes, capturing intuitive episodes, measuring individual differences in intuitive processing, and identifying the neural correlates of intuitive processes and outcomes (Hodgkinson \& Sadler-Smith, 2011). From a dual-process perspective there are a number of valid and reliable instruments for the self-report measurement of individual differences in preferences for intuition in general (Betsch, 2008; Epstein et al., 1996). The delineation of different types of intuition raises new challenges and opportunities, namely: examining relationships between extant measures of intuition; designing, developing, and validating more bespoke psychometric tools over-and-above individual differences in preferences for intuition, and for assessing intuition in its different guises. Indeed, it may be advisable for intuition researchers to focus also on the assessment of performance in the various domains of intuition using task-based measures, for example, ethical and moral dilemmas or tacit knowledge tests (see Haidt, 2001; Wagner \& Sternberg, 1985).

A more systematic program of phenomenological research might provide insights into the subjective nature experiences of intuition, using techniques such as critical incident technique (Flanagan, 1954), applied cognitive task analysis (Gore \& McAndrew, 2009) experience sampling method (Csikszentmihalyi \& Larsen 1987) (see Hodgkinson, Langan- 
Fox, \& Sadler-Smith, 2008). The elicitation of first-person accounts of intuitive episodes might also deploy the techniques of guided introspection based on psycho-phenomenological methods which have the potential to offer increased 'granularity' in the data (Vermersch, 1999), and the analysis of primary metaphors primary metaphors that have a sensory-motor grounding in the body (Feldman, 2008). Significant advances have been made in recent years in the use of brain imaging and this line of research is likely to continue to gain in momentum and make greater inroads into the study of intuitive processing in applied fields and occupational settings. It is through the convergence of complementary lines of evidence from the behavioural, information processing, and neural levels of analysis that greatest progress is likely to be achieved (Akinci \& Sadler-Smith, 2012).

The study of intuition has progressed to the extent that a number of mechanisms and processes of intuiting are identifiable, and as relevant developments in the evolutionary, brain, and behavioural sciences continue apace, the basic science upon which intuition researchers are able to draw will strengthen. Much is to be gained through inter-disciplinary biological, social, and cultural inquiry, and intuition researchers can look forward to evidence for other processes and types of intuition emerging through on-going research in laboratory and field settings. An improved understanding of the workings of a complex system of intuitive judgment and reasoning founded upon the extant and emerging evidence base from within the behavioural, brain, and evolutionary sciences has much to offer the applied study of intuitive decision processes in laboratory and organizational field settings. 


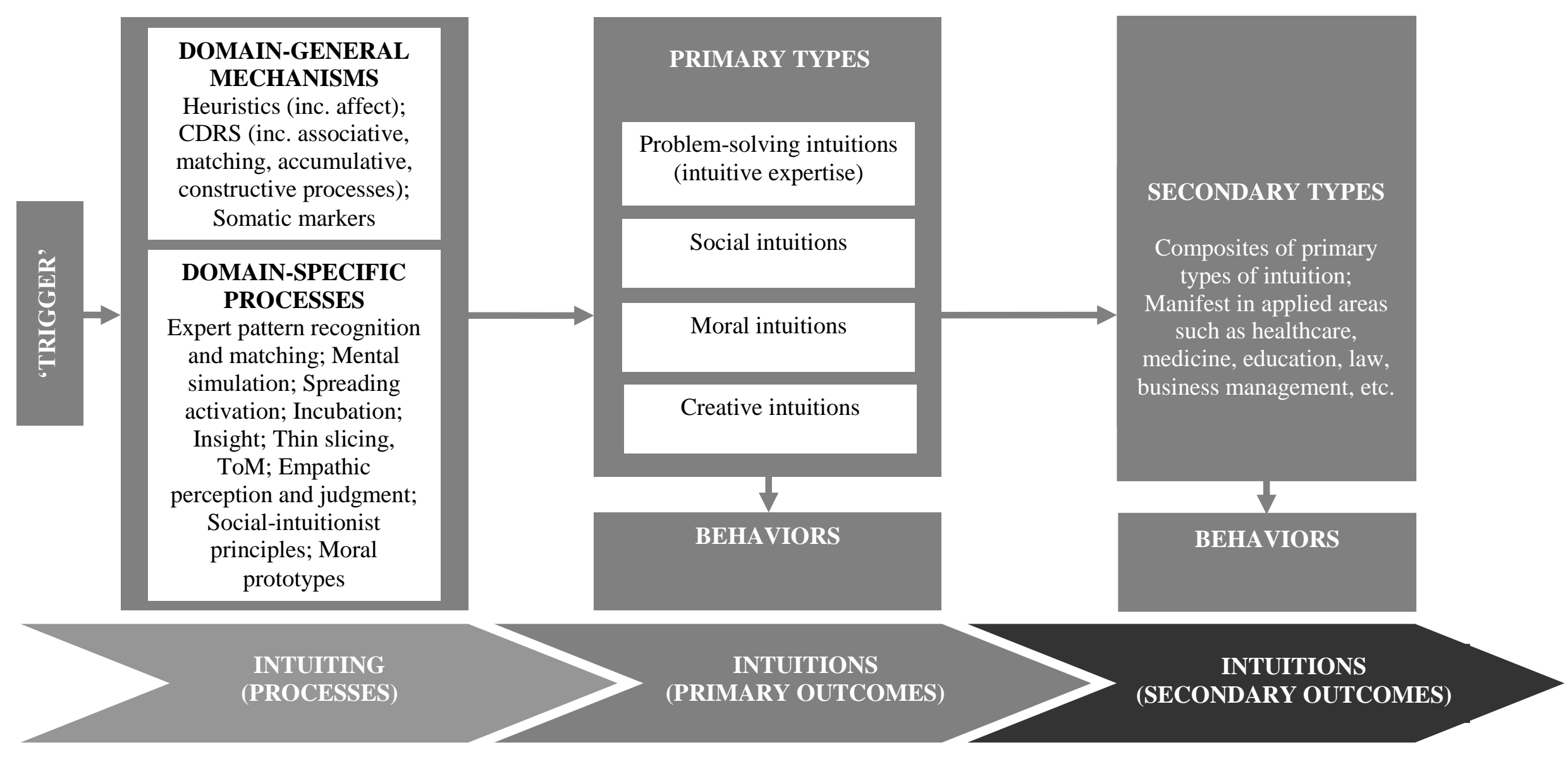

Figure 1. Conceptual framework (Note: CDRS, complex domain-relevant schemas; ToM, theory of mind) 
Table 1.

Distinctions between and within intuiting and intuition

Intuiting Domain-general cognitive and affective mechanisms of intuiting

Domain-specific processes

Intuitions Primary types of intuition

Secondary types
Fundamental aspects of human learning, reasoning and judgment which operate across domains;

Evoked automatically on the basis of the specific characteristics (e.g. complexity, riskiness and uncertainty) of the eliciting task (i.e. the 'trigger' for non-deliberative processing);

1. Build complex domain-relevant schemas (via explicit and implicit learning);

2. Provide affective 'data' (e.g. manifest as somatic markers);

3. Give rise to rapid judgments which may be subject to intuitive errors (on the basis of heuristics and biases).

Activated autonomously on the basis of:

1. Characteristics of recurrent (i.e. more frequently encountered) domains in which the task is located;

2. Learned patterns, schemas and prototypes

Outcomes of intuiting reducible only to information processing mechanisms and their neural correlates in the recurrent domains of:

1. Problem-solving;

2. Creativity;

3. Moral judgment;

4. Social judgment

Composite forms of intuition; analyzable in terms of and reducible to, primary types; deployed in specific and nonrecurrent (i.e. less frequently encountered) domains in occupational settings, e.g. entrepreneurial intuition in business management 
Table 2.

Primary types of intuition: behavioral, information processing and neural descriptions

\section{Primary Behavioral Description Type}

Problemsolving intuition

Creative intuition intuition

Moral intuition to a creative outcome.
Domain-specific, expertise-based response to a tightly-structured problem based on non-conscious processing of information, activated automatically, eliciting matching of complex patterns of multiple cues against previously acquired prototypes and scripts held in long-term memory. Slow-to-form affectively-charged judgment occurring in advance of an insight that combines knowledge in novel ways based on divergent associations, and which orients behavior in a direction that may lead

Rapid and automatic evaluation of another person's cognitive and/or affective state through the perception and non-conscious processing of verbal and/or non-verbal indicators. Automatic, rapid, affect-based judgment made in response to an ethical dilemma, arrived at nonconsciously, rationalized post-hoc, and relatively impervious to disconfirmation.

\section{Information \\ Processing Mechanisms}

Pattern Orbito-frontal recognition and matching; Action scripts; Mental simulation

\section{Neural correlates} cortex; Ventral occipito-temporal regions; Posterior hippocampus.

Spreading

Diffuse neural activation; networks; Anterior Incubation; Insight superior temporal gyrus

Thin slicing; Lateral temporal Theory of Mind; cortex; Von Empathic Economo Neurons; perception and Mirror neurons judgment

Social-intuitionist principles; Moral prototypes

Ventro-medial prefrontal cortex and related $\mathrm{X}$ system substrates 


\section{References}

Adolphs, R. \& Damasio, A. (2001). The interaction of affect and cognition: A neurobiological perspective. In J. Forgas (Ed.) Handbook of affect and social cognition. Hillsdale, NJ: Erlbaum, 27-49.

Akinci, C. \& Sadler-Smith, E. (2012). Intuition in management research: A historical review. International Journal of Management Reviews, 14 (in press).

Allinson, C. W. and Hayes, J. (1996). The Cognitive Style Index: A measure of intuitionanalysis for organizational research. Journal of Management Studies, 33(1), 119-135.

Allinson, C.W. Chell E., \& Hayes, J. (2000). Intuition and entrepreneurial performance. European Journal of Work and Organizational Psychology, 9, 31-43.

Allman, J.M., Watson, K.K., Tatreault, N.A., \& Hakeem, A.Y. (2005). Intuition and autism: A possible role for Von Economo neurons. Trends in Cognitive Sciences, 9(8), 367-373.

Almor, A. (2003). Specialized behavior without specialized modules. In D.E. Over (Ed.) Evolution and the psychology of thinking: The debate, 101-120. Hove: Psychology press. Ambady, N. (2010). The perils of pondering: Intuition and thin slice judgments. Psychological Inquiry, 21(4), 271-278.

Ambady, N. \& Krabbenhoft, M.A. (2006). The 30-sec sale: Using thin slice judgments to evaluate sales effectiveness. Journal of Consumer Psychology, 16(1), 4-13

Ambady, N. \& Rosenthal, R. (1993). Half a minute: Predicting teacher evaluations from thin slices of nonverbal behavior and physical attractiveness. Journal of Personality and Social Psychology, 64, 431-441.

Ambady, N., Koo, J.J., Rosenthal, R., \& Winograd, C. (2002). Physical therapists’ nonverbal communication predicts geriatric patients' health outcomes. Psychology and Aging, $17,443-452$ 
Ambady, N., La Plante D., Nguen, T., Rosenthal, R., \& Levinson, W. (2002). Surgeon's tone of voice: A clue to malpractice history. Surgery, 132, 5-9

Andrews, P.W. \& Thomson, J.A. (2009). The bright side of being blue: Depression as an adaptation for analyzing complex problems. Psychological Review, 116(3), 620-654.

Bar, M. et al. (2006). Top-down facilitation of visual recognition. Proceedings of the National Academy of Sciences, 103, 449-454.

Bargh, J.A. \& Chartrand, T.L. (1999). The unbearable automaticity of being. American Psychologist, 54, 462-79.

Bechara, A. (2004). The role of emotion in decision making: Evidence from neurological patients with orbito-frontal damage. Brain and Cognition, 55, 30-40.

Bechara, A. \& Damasio, A.R. (2005). The somatic marker hypothesis: A neural theory of economic decision. Games and Economic Behavior, 52, 336-372.

Betsch, C. (2008). Chronic preferences for intuition and deliberation in decision making: Lessons learned about intuition from an individual differences approach, In Plessner, H., Betsch, C. and Betsch, T. (Eds.) Intuition in judgment and decision making (pp. 231-248). New York: Lawrence Erlbaum Associates.

Blume, B.D. \& Covin, J.G. (2010). Attributions to intuition in the venture founding process: Do entrepreneurs use intuition or just say that they do? Journal of Business Venturing, 26, $137-151$.

Bolte, A., Goschke, T. \& Kuhl, J (2003). Emotion and intuition: Effects of positive and negative mood on implicit judgments and semantic coherence. Psychological Science, $14(5), 416-421$.

Bowers, K. S., Regehr, G., Balthazard, C., \& Parker, K. (1990). Intuition in the context of discovery. Cognitive Psychology, 22, 72-110. 
Brainerd, C.J. \& Reyna, V.F. (1990). Gist is the grist: Fuzzy-trace theory and the new intuitionism. Developmental Review, 10, 3-47.

Buchholz, R.A. \& Rosenthal, S.B. (2005). The spirit of entrepreneurship and the qualities of moral decision making: Toward a unifying framework. Journal of Business Ethics, 60, 307-315.

Campitelli, G. \& Gobet, F. (2010). Herbert Simon’s decision-making approach: Investigation of cognitive processes in experts. Review of General Psychology, 14(4), 354-364.

Campitelli, G., Gobet, F., \& Parker, A. (2005). Structure and stimulus familiarity: A study of memory in chess players with fMRI. Spanish Journal of Psychology, 8(2), 238-245.

Campitelli, G., Gobet, F., Head, K., Buckley, M., \& Parker, A. (2007) Brain localization of memory chunks in chess players. International Journal of Neuroscience, 117(12), 16411659.

Cappon, D. (1994). Intuition and management: Research and application. Westport: Quorum Books

Chiappe, D. \& MacDonald, K. (2005). The evolution of domain-general mechanisms in intelligence and learning. The Journal of General Psychology, 132(1), 5-40.

Claxton, G. (2001). The anatomy of intuition. In T. Atkinson, T., \& G. Claxton, (Eds). The intuitive practitioner (pp.32-52). Buckingham: Open University Press.

Claxton, G. (2006). Thinking at the edge. Cambridge Journal of Education, 36(3), 351-362.

Crossan, M.M., Lane, H.W., \& White, R.E. (1999). An organizational learning framework: From intuition to institution. Academy Management Review, 24, 522-537.

Csikszentmihalyi M. \& Larsen R. (1987). Validity and reliability of the experiencesampling method. Journal of Nervous and Mental Disorders, 175, 526-36. 
Cushman, R, Young, L., \& Hauser, M. (2006). The role of conscious reasoning and intuition in moral judgment. Psychological Science, 17, 1082-89.

Damasio, A.R. (1996). The somatic marker hypothesis and the possible functions of the prefrontal cortex. Philosophical Transactions of the Royal Society of London B, 351, 1413-1420.

Damasio, A.R. (1999). The feeling of what happens: Body, emotion and the making of consciousness. London: Vintage.

Dane, E. \& Pratt, M.G. (2007). Exploring intuition and its role in managerial decision making. Academy of Management Review, 32(1), 33-54.

Dane, E. \& Pratt, M.G. (2009). Conceptualizing and measuring intuition: A review of recent trends. In G.P. Hodgkinson and J.K. Ford (Eds.), International Review of Industrial and Organizational Psychology - Volume 24 (pp.1-40). Wiley: Chichester, UK

Davidson, J.E. (1995). The suddenness of insight. In R.J. Sternberg \& J.E. Davidson (Eds.) The nature of insight (pp.125-155). Cambridge, MA.: The MIT Press.

De Martino, B., Kumaran, D., Seymour, B., \& Dolan, R.J. (2006). Frames, biases, and rational decision-making in the human brain. Science, 313(5787), 684-687.

DePaulo, B.M. (1992). Nonverbal behavior and self-presentation. Psychological Bulletin, $111(2), 203-243$.

Dijksterhuis, A. (2004). Think different: The merits of unconscious thought in preference development and decision making. Journal of Personality and Social Psychology, 87, 586-598.

Dorfman, J., Shames, V.A. \& Kihlstrom, J.F. (1996). Intuition, incubation and insight: implicit cognition and problem solving. In G. Underwood (Ed.) Implicit cognition, 257296. Oxford: Oxford University Press.

Duggan, W. (2007). Strategic intuition. New York: Columbia University Press. 
Dunn, B.D., Dalgleish, T. and Lawrence, A.D. (2006) The somatic marker hypothesis: A critical evaluation. Neuroscience and Behavioral Reviews, 30, 239-271.

Epstein, S. (2008) "Intuition From the Perspective of Cognitive-Experiential Self-Theory" In Plessner, H., Betsch, C. and Betsch, T. (Eds.) Intuition in Judgment and Decision Making (pp.23-37). New York: Taylor \& Francis Group, LLC.

Epstein, S., Pacini, R., Denes-Raj, V., \& Heier, H. (1996). Individual differences in intuitive-experiential and analytical-rational thinking styles. Journal of Personality and Social Psychology, 71, 390-405.

Ericsson K.A. \& Charness. N. (1994). Expert performance: Its structure and acquisition. American Psychologist, 49, 725-747.

Evans, J St.B.T. (2003). In two minds: Dual-process accounts of reasoning. Trends in Cognitive Sciences, 7(10), 454-459.

Evans, J. St., B. T. (2008). Dual-processing accounts of reasoning, judgment, and social cognition. Annual Review of Psychology, 59, 6.1-6.24.

Fazio, R. H., \& Towles-Schwen, T. (1999). The MODE Model of attitude-behavior processes. In S. Chaiken \& Y. Trope (Eds.), Dual-process theories in social psychology (pp. 97-116). New York: Guilford Press.

Feldman, J.A. (2008). From molecule to metaphor: A neural theory of language. Cambridge, MA.: MIT Press

Fiedler, K. (2001). Affective influences on social information processing, In Forgas 163-185 Finucane, M.L., Alhakami, A., Slovic, P., \& Johnson, S.M. (2000). The affect heuristic in judgment of risks and benefits. Journal of Behavioral Decision Making, 13, 1-17.

Flanagan, J.C. (1954). The critical incident technique. Psychological Bulletin, 51, 327-358. Forgas, J.P. (2001). The affect infusion model. In L.L. Martin \& G.L. Clore (Eds.) Theories of mood and cognition (pp.99-134). Mahwah, NJ.: LEA. 
Friedman, R.S., \& Förster, J. (2005). Effects of motivational cues on perceptual asymmetry. Journal of Personality and Social Psychology, 88(2), 263-275.

Frith, U. \& Happe, F. (1994) Autism: beyond "theory of mind". Cognition, 50, 115-132.

Garronkski, B. \& Bodenhausen, G.V. (2007). Unravelling the processes underlying evlaluation: Attitudes fpom the perspective of the APE model. Social Cognition, 25, 687717

George, J.M. \& Zhou, J. (2007). Dual tuning in a supportive context: joint contributions of positive mood, negative mood, and supervisory behaviors to employee creativity. Academy of Management Journal, 50(3), 605-622.

Gick, M.L. \& Lockhart, R.S. (1995). Cognitive and affective components of insight. In R.J. Sternberg \& J.E. Davidson (Eds.) The nature of insight (pp.197-228). Cambridge, MA.: The MIT Press.

Gigerenzer, G. \& Brighton, H. (2009). Homo heuristicus: Why biased minds make better choices. Topics in Cognitive Science, 1, 107-143.

Gilovich, T. and Griffin, D. (2002) Introduction - heuristics and biases: Then and now. In T., Gilovich, D. Griffin, and D. Kahneman, (Eds.) (2002) Heuristics and Biases: The Psychology of Intuitive Judgment (pp. 1-18). New York: Cambridge University Press.

Glöckner, A. \& Witteman, C. (2010). Beyond dual-process models: A categorization of processes underlying intuitive judgment and decision making. Thinking and Reasoning, $16(1), 1-25$.

Goldsmith, R.E. (1994). Creative style and personality theory. In M.J. Kirton (Ed.) Adaptors and innovators: Styles of creativity and problem solving, 34-50. London: Routledge.

Gore. J. and McAndrew, C. (2009) Accessing expertise. The Psychologist. 22 (3), 218-219 
Gore, J., Banks, A., Millward, L., \& Kyriakidou, O. (2006). Naturalistic decision making: reviewing pragmatic science. Special Issue: Naturalistic Decision making in organizations. Organization Studies, 27(7), 925-942.

Greene, J., \& Haidt, J. (2002). How (and where) does moral judgment work? Trends in Cognitive Sciences, 16, 517-519.

Gruber, H. (1995) Insight and affect in the history of science. In R.J. Sternberg \& J.E. Davidson (Eds.) The nature of insight (pp.397-431). Cambridge, MA.: The MIT Press.

Haidt, J. (2001). The emotional dog and its rational tail: A social intuitionist approach to moral judgment. Psychological Review, 108, 814-834.

Haidt, J. (2004). The emotional dog gets mistaken for a possum. Review of General Psychology, 8(4), 283-290.

Harvey, J. (1999). Music and inspiration. London: Faber and Faber.

Hauser, M.D. (2006). Moral minds. London: Abacus.

Helie, S., \& Sun, R. (2010). Incubation, Insight, and Creative Problem Solving: A Unified Theory and Connectionist Model. Psychological Review, 117(3), 994-1024

Hodgkinson, GP., Langan-Fox, J., \& Sadler-Smith, E. (2008). Intuition: A fundamental bridging construct in the behavioural sciences. British Journal of Psychology, 99(1), 1-27.

Hodgkinson, G. P. \& Sadler-Smith, E. (2003) Complex or unitary? A critique and empirical re-assessment of the Allinson-Hayes Cognitive Style Index. Journal of Occupational and Organizational Psychology, 76, 243-268.

Hodgkinson, G.P. \& Sadler-Smith, E. (2011). Investigating intuition: Beyond self-report. In M. Sinclair (ed.) Handbook of Intuition Research, Edited by M. Sinclair, Cheltenham, UK: Elgar (in press). 
Hodgkinson, G.P., Sadler-Smith, E., Sinclair, M. \& Ashkanasy, N. (2009) More than meets the eye? Intuition and analysis revisited. Personality and Individual Differences, 47, 342346.

Hogarth, R.M. (2001). Educating intuition. Chicago: University of Chicago Press.

Hogarth, R.M. (2010). Intuition: A challenge for psychological research on decision making. Psychological Inquiry, 21(4), 338-353.

Hunt, R.G., Krzystofiak, F.J., Meindl, J.R., \& Yousry, A.M. (1989). Cognitive style and decision making. Organizational Behavior and Human Decision Processes, 44(3), 436453.

Jausovec, N. \& Bakracevic, K. (1995). What can heart rate tell us about the creative process? Creativity Research Journal, 8(1), 11-24.

Jung-Beeman M., Bowden, E.M., Haberman, J., Frymiare, J., Arambel-Lui, S., Greenblat, R., Reber, P.J. \& Kounios, J. (2004a). Neural activity when people solve problems with insight. Public Library of Science (Biology), 2(4), 0500-0510.

Kahneman, D. (2002). Maps of bounded rationality: a perspective in intuitive judgment and choice. Nobel Prize Lecture on 8 December 2002, 449-489. Available from nobelprize.org/nobel_prizes/economics/laureates/2002.

Kahneman, D. \& Frederick, S. (2002). Representativeness revisited: attribute substitution in social judgment. In Gilovich, T., Griffin, D., \& Kahneman, D. (Eds.) Heuristics and biases: the psychology of intuitive judgment (pp.49-81). New York: Cambridge University Press.

Kahneman, D. \& Frederick, S. (2005). A model of heuristic judgment. In K. Holyoak, \& R.G. Morrison (Eds). The Cambridge handbook of thinking and reasoning (pp.267-294). Cambridge, UK: Cambridge Univ. Press. 
Kahneman, D. \& Klein, G.A. (2009). Conditions for intuitive expertise: a failure to disagree. American Psychologist, 64(6), 515-526.

Kahneman, D., Slovic, P. \& Tversky, A. (Eds.). (1982). Judgment under uncertainty: Heuristics and biases, New York: Cambridge University Press.

Kaufman, J.C. \& Baer, J.M. (2009). How are intellectual styles related to creativity across multiple domains? In L-F. Zhang \& R.J. Sternberg (Eds.) Perspectives on the nature of intellectual styles (pp.87-106). New York: Springer.

Kickul, J., Gundry, L.K., Barbosa, S.D., \& Whitcanack, L. (2009) Intuition Versus Analysis? Testing Differential Models of Cognitive Style on Entrepreneurial Self-Efficacy and the New Venture Creation Process. Entrepreneurship Theory and Practice, 439-453.

Kirton, M.J. (1976). Adaptors and innovators: A description and measure. Journal of Applied Psychology, 61, 622-629

Klein, G.A. (1993). A recognition-primed decision (RPD) model of rapid decision making. In G.A. Klein, J. Orasanu, R. Calderwood, \& C.E. Zsambok (Eds.). Decision making in action: Models and methods (pp.138-147). Norwood, CT.: Ablex.

Klein, G.A. (2003). Intuition at work. New York: Doubleday.

Klein, G.A., Wolf, S., Militello, L., \& Zsambok, C. (1995). Characteristics of skilled option generation in chess. Organizational Behavior and Human Decision Processes, 62(1), 6369.

Koriat, A. (1994). How do we know that we know? The accessibility model of the feeling of knowing. Psychological Review, 100(4), 609-639.

Kruglanski, A.W. \& Orehek, R. (2007). Partitioning the domain of social inference: Dual mode and systems models and their alternatives. Annual Review of Psychology, 58, 291316.

Lakoff, G. \& Johnson, M. (1999). Philosophy in the flesh. New York: Basic Books. 
LeDoux, J. E. (1996) The Emotional Brain: The Mysterious Underpinnings of Emotional Life, New York: Simon and Schuster.

Lieberman, M.D. (2000). Intuition: A social cognitive neuroscience approach. Psychological Bulletin, 126, 109-137.

Lieberman, M.D. (2005). Principles, processes, and puzzles of social cognition. NeuroImage, 28, 745-756.

Lieberman, M.D. (2007). Social cognitive neuroscience: A review of core processes. Annual Review of Psychology, 58, 259-289.

Lieberman, M (2009). What zombies can't do. In Evans, J. St. B. T. \& Frankish, K. (Eds.) In two minds: Dual-processes and beyond (pp.293-315). Oxford: Oxford University Press.

Lieberman, M.D., Jarcho, J.M., \& Satpute, A.B. (2004). Evidence-based and intuition-based self-knowledge: An fMRI study. Journal of Personality and Social Psychology, 87, 421435.

Lipshitz, R., Klein, G.A., Orasanu, J., \& Salas, E. (2001). Taking stock of naturalistic decision making. Journal of Behavioral Decision Making, 14, 331-352.

Maguire, E.A., Woollett, K., \& Spiers, H.J. 2006. London taxi drivers and bus drivers: A structural MRI and neuropsychological analysis. Hippocampus, 16, 1091-1101.

Marquardt, N. \& Hoeger, R. (2009). The effect of implicit moral attitudes on managerial decision making: An implicit social cognition approach. Journal of Business Ethics, 85, $157-171$.

Metcalfe J. \& Wiebe, D. (1987). Intuition in insight and non-insight problem-solving. Memory and Cognition, 15(3), 238-246.

Metcalfe, J. \& Mischel, W. (1999). A hot/cool-system analysis of delay of gratification: Dynamics and willpower. Psychological Review, 106, 3-19. 
Mikhail, J. (2007). Universal moral grammar: Theory, evidence and the future. Trends in Cognitive Sciences, 11, 143-152.

Miller, C.C. \& Ireland, R.D. (2005). Intuition in strategic decision making: Friend or foe in the fast-paced 21st Century? Academy of Management Executive, 19(1), 19-30.

Mithen, S. (1996). The prehistory of the mind: The search for the origins of art, religion, and science. London: Phoenix.

Monin, B., Pizzaro, D.A., \& Beer, J.S. (2007). Deciding versus reacting: Conceptions of moral judgment and the reason-affect debate. Review of General Psychology, 11(2), 99111.

Myers, D.G. (2002). Intuition: Its powers and perils. New Haven and London: Yale University Press.

Nadler, J., Thompson, L. \& van Boven, L. (2003). Learning negotiation skills: four models of knowledge creation and transfer. Management Science, 49(4), 9-540

Nicholson, N. 2000. Managing the human animal. London: Texere

Nisbett, RE, \& Ross, L. (1980). Human inference: Strategies and shortcomings of social judgment. Englewood Cliffs, NJ: Prentice-Hall

Nisbett, R.E., \& Wilson, T.D. (1977). Telling more than we can know: verbal reports on mental processes. Psychological Review, 84, 231- 259.

Oberman, L.M. \& Ramachandran, V.S. (2007). The simulating social mind: The role of the mirror neuron system and simulation in the social and communicative deficits of autism spectrum disorders. Psychological Bulletin, 133(2), 310-327.

Pacini, R. \& Epstein S. (1999). The relation of rational and experiential information processing styles to personality, basic beliefs, and the ratio-bias phenomenon. Journal of Personality and Social Psychology, 76, 972-987. 
Payne, B.K. (2001). Prejudice and perception: The role of automatic and controlled processes in misperceiving a weapon. Journal of Personality and Social Psychology, 81, 181-192.

Plessner, H., Betsch, T., Schallies, E., \& Schwieren, C. (2008) “Automatic Online Formation of Implicit Attitudes Towards Politicians as a Basis for Intuitive Voting Behavior” In Plessner, H., Betsch, C. \& Betsch, T. (Eds.) Intuition in Judgment and Decision Making (pp.107-117). New York: Lawrence Erlbaum Associates.

Policastro, E. (1995). Creative intuition: An integrative review. Creativity Research Journal, 8(2), 99-113.

Policastro, E. \& Gardner, H. (1999). From case studies to robust generalizations: An approach to the study of creativity. In R.J. Sternberg (Ed.) Handbook of Creativity, 213224, Cambridge: Cambridge University Press.

Porter, S., Doucette, N.L., Woodworth, M., Earle, J. \& MacNeil, B. (2008). Halfe the world knowes not how the other halfe lies: Investigation of verbal and non-verbal signs of deception exhibited by criminal offenders and non-offenders. Legal and Criminal Psychology, 13, 27-38.

Reynolds, S.J. (2006). A neuro-cognitive model of the ethical decision making process: Implications for study and practice. Journal of Applied Psychology, 91, 737-748.

Rizzolatti, G. \& Craighero, L. (2004). The mirror-neuron system. Annual Review of Neuroscience, 27, 169-192.

Sadler-Smith, E. (2008). Inside intuition. Abingdon: Routledge.

Sadler-Smith, E. (2010). The intuitive mind. Chichester: John Wiley and Sons.

Sadler-Smith, E., Hodgkinson, G. P., \& Sinclair, M. (2008). A matter of feeling? The role of intuition in entrepreneurial decision making and behavior. In W. J. Zerbe, C. E. J. Hartel, \& N. M. Ashkanasy (Eds.), Research on emotion in organizations: Emotions, ethics and decision-making, Volume 4 (pp.35-55). Bingley, UK: Emerald JAI. 
Salas, E. \& Klein, G.A. (2001). Expertise and naturalistic decision making: An overview. In E. Salas \& G.A. Klein (Eds.) Linking expertise and naturalistic decision making (pp.38). Mahwah, N.J.: Lawrence Erlbaum.

Salas, E., Rosen, M.A. \& DiazGranados, D. (2010) Expertise-based intuition and decision making in organizations. Journal of Management, 36, 941-973.

Saltzstein, H.D. \& Kasachkoff, T. (2004). Haidt's moral intuitionist theory: A psychological and philosophical critique. Review of General Psychology, 8(4), pp.273-282.

Salvador, R. \& Folger, R.G. (2009). Business ethics and the brain, Business Ethics Quarterly, 19(1), 1-31.

Satpute A.B. \& Lieberman, M.D. (2006). Integrating automatic and controlled processing into neurocognitive models of social cognition. Brain Research, 1079, 86-97.

Saxe, R., Carey, S., \& Kanwisher, N. (2004). Understanding other minds: Linking developmental psychology and functional neuroimaging. Annual Review of Psychology, $55,87-124$.

Schneider, W. \& Shiffrin, R.M. (1977). Controlled and automatic human information processing: I. Detection, search, and attention. Psychological Review, 84, 1-66.

Schulte-Rüther, M., Markowitsch, H.J., Fink, G.R., \& Piefke, M. (2007). Mirror neuron theory of mind mechanisms involved in face-to-face interaction. Journal of Cognitive Neuroscience, 19(8), 1354-1372.

Seo, M.G., Barrett, L.F., \& Bartunek, J.M. (2004). The role of affective experience in work motivation. Academy of Management Review, 29(3), 423-439.

Shane, S. \& Venkataraman, S (2000). The promise of entrepreneurship as a field of research. Academy of Management Review, 25(1), 217-226. 
Sherman, J.W., Gawronski, B., Gonsalkorale, K., Hugenberg, K., Allen, T.J., \& Groom, C.J. (2008) The self-regulation of automatic associations and behavioral impulse. Psychological Review, 115, 314-335.

Simon, H.A. (1987). Making management decisions: The role of intuition and emotion. Academy of Management Executive, 1(1), 57-64.

Simon, H.A. \& Chase, W.G. (1973). Skill in chess. American Scientist, 61, 393-403.

Simonton, D.K. (1980). Intuition and analysis: A predictive and explanatory model, Genetic Psychology Monographs, 102, 3-60.

Sinclair, M. (2010). Misconceptions about intuition. Psychological Inquiry, 21(4), 378-386.

Singer, T. (2006). The neuronal basis and ontogeny of empathy and mind reading: review of literature and implications for future research. Neuroscience \& Biobehavioral Reviews, $30(6), 855-863$.

Slovic, P., Finucane, M. L., Peters, E., \& MacGregor, D.G. (2004). Risk as analysis and risk as feelings: Some thoughts about affect, reason, risk, and rationality. Risk Analysis, 24 (2), 311-322.

Smith, E.R. \& DeCoster, J. (2000). Dual-process models in social and cognitive psychology: Conceptual integration and links to underlying memory systems. Personality and Psychology Review, 4, 108-131.

Sonenschein, S. (2007). The Role of construction, intuition, and justification in responding to ethical issues at work: The sensemaking-intuition model. Academy of Management Review, 32(4), 1022-1040.

Stanovich, K.E. \& West, R.F. (2000). Individual differences in reasoning: Implications for the rationality debate? Behavioral and Brain Sciences, 23, 645-65.

Sternberg R.J. \& Davidson J.E. (1995). The nature of insight Cambridge, MA.: The MIT Press. 
Sundgren, M. \& Styhre, A. (2004). Intuition and pharmaceutical research: The case of AstraZeneca. European Journal of Innovation Management, 7(4), 267-279.

Toplak, M.E., Liu, E., Macpherson, R., Toneatto, T., \& Stanovich, K.E. (2007). The reasoning skills and thinking dispositions of problem gamblers: A dual-process taxonomy. Journal of Behavioral Decision Making, 20, 103-124.

Vaughan, F.E. (1979). Awakening intuition. New York: Doubleday.

Vermersch, P. (1999). Introspection as practice. Journal of Consciousness Studies, 6(2/3), $17-42$.

Volz, K.G. \& von Cramon, D.Y. (2006). What neuroscience can tell about intuitive processes in the context of perceptual discovery. Journal of Cognitive Neuroscience, 18(12), 2077-2087.

Wagner, R.K. \& Sternberg, R.J. (1985). Practical intelligence in real-world pursuits: The role of tacit knowledge, Journal of Personality and Social Psychology, 48, 436-458.

Wallas. G. (1926). The art of thought. New York: Franklin Watts.

Wild, K.E. (1938). Intuition. Cambridge: Cambridge University Press.

Wilson, T.D., Lindsay, S., \& Schooler, T.Y. (2000). A model of dual attitudes. Psychological Review, 107(1), 101-126.

Yaniv, I., \& Meyer, D. E. (1987). Activation and metacognition of inaccessible stored information: Potential bases for incubation effects in problem solving. Journal of Experimental Psychology: Learning, Memory, and Cognition, 13(2), 187-205.

Young L. \& Koenigs, M. (2007). Investigating emotion in moral cognition: A review of evidence from functional neuro-imaging and neuropsychology. British Medical Bulletin, 84(1), 69-79. 
Zhang, L-F. \& Sternberg, R.J. (2009). Revisiting the value issue in intellectual styles, In LF. Zhang \& R.J. Sternberg (Eds.) Perspectives on the nature of intellectual styles (pp.6385). New York: Springer. 